\title{
Formen und Ausmaß verstärkter Arbeitszeitflexibilisierung
}

Flexible Formen der Arbeitszeit haben in den letzten Jahren enorm an Bedeutung gewonnen und bestimmen mittlerweile den betrieblichen Alltag. Diese Entwicklung hat eine neue Stufe erreicht. Verschiedene Formen flexibler Arbeitszeit überlagern und verstärken sich. Welches Ausmaß multiple Arbeitszeitflexibilität erreicht hat und welche Beschäftigtengruppen davon betroffen sind, untersucht der folgende Beitrag.

\section{Einleitung}

Die Arbeitszeit vollzieht einen tief greifenden Wandel. Dauer, Lage und Verteilung folgen immer weniger dem Muster der Normalarbeitszeit. Die Arbeitszeit wird nicht nur kürzer, sondern auch länger und zugleich flexibler. Ein wachsender Teil der Beschäftigten arbeitet im Rahmen von Teilzeit oder Mini-Jobs deutlich unter dem Niveau der tariflichen Regelarbeitszeiten. Genau in die andere Richtung bewegt sich die Arbeitszeit für die schrumpfende Zahl der Vollzeitbeschäftigten. Deren durchschnittliche effektive und in Teilbereichen auch tarifliche Arbeitszeit nimmt wieder zu. In dieser Zeitenwende spiegelt sich der seit Jahren anhaltende Druck der Betriebe wider, im internationalen Standortwettbewerb die Arbeitskosten zu reduzieren. Im Tausch gegen Beschäftigungssicherungen haben Arbeitnehmer und Gewerkschaften längere und flexiblere Arbeitszeiten akzeptieren müssen.

Überlagert wird der Trend zu polarisierten Arbeitszeiten von der anhaltenden Tendenz zu atypischen Arbeitszeiten. Nacht- und Wochenendarbeit verlieren ihren Ausnahmecharakter und werden schrittweise zur „neuen Normalität“. Am stärksten hat sich das Verteilungsmuster der Arbeitszeit gewandelt. Arbeitszeitkonten haben für einen Modellwechsel gesorgt. Sie erlauben, die Arbeitszeit variabel zu verteilen und lösen das lange Zeit dominierende Muster gleichförmiger Arbeitszeiten ab. Die Normalarbeitszeit verliert ein konstitutives Merkmal.

Dieser Umbruch lässt sich nur adäquat erfassen, wenn man die Entwicklungen der drei Dimensionen der Arbeitszeit simultan betrachtet und analysiert. Dieser Beitrag macht einen Schritt in diese Richtung und versucht, die bislang in der Literatur vorherrschende eindimensionale Analyseperspektive zu erweitern. Die nachfolgende Untersuchung will aufzeigen, ob und wie sich die anhand der genannten Dimensionen vorgenommenen Flexibilisierungen der Arbeitszeit miteinander verschränken. Der Beitrag skizziert zunächst die normgebenden Funktionen der Normalarbeitszeit (Abschnitt 2), bevor einige Untersuchungshypothesen formuliert werden (Abschnitt 3). In Abschnitt 4 folgt eine multivariate Analyse, die die Normalarbeitszeit extremen Formen flexibler Arbeitszeit gegenüberstellt.

\section{Norm und Normalität der Arbeitszeit}

Die sogenannte Normalarbeitszeit dient üblicherweise als Referenzgröße, um Veränderungen in den Grundmustern der Arbeitszeit zu identifizieren. Neben ihrer analytischen hat sie eine normative Funktion. Außerdem galt sie lange Zeit als das dominierende Modell für die Gestaltung von Dauer, Lage und Verteilung der Arbeitszeit. Abweichungen hatten eher Ausnahmecharakter, waren begründungspflichtig und meistens auch mit einer zusätzlichen Vergütung verbunden. Die Zeiten haben sich geändert. Flexible Arbeitszeitmuster haben die Oberhand gewonnen. Traditionell atypische Arbeitszeiten (Schicht-, Nacht- und Wochenendarbeit) sind zur „neuen Normalität" geworden (Seifert 2007). Noch mehr gilt dies für variable Arbeitszeitfor- men auf Basis von Zeitkonten. Schließlich ist auch Teilzeitarbeit für viele Frauen eine normale Arbeitszeit.

Angesichts dieses Modellwechsels hin $\mathrm{zu}$ flexiblen und heterogenen Arbeitszeiten sowie der verblassenden empirischen Relevanz der Normalarbeitszeit stellt sich die Frage, ob damit auch deren normgebende Funktion verloren geht. Das hätte weitreichende Konsequenzen für die Bewertung unterschiedlicher Arbeitszeiten. Hinsichtlich ihrer Dauer, Lage und Verteilung divergierende Arbeitszeiten würden ungeachtet ihrer jeweiligen ökonomischen Bedeutung und ungeachtet der Belastungen und Beeinträchtigungen für die Beschäftigten als gleichwertig angesehen und behandelt. Während die Arbeitszeiten in ihren Formen heterogener werden, würden sie in ihrer Bewertung homogener.

Bevor wir der Frage nachgehen, welche Auswirkungen die abnehmende empirische Bedeutung der Normalarbeitszeit auf ihre normgebende Funktion hat, ist die Ab-

Hermann Groß, Dr., wissenschaftlicher
Angestellter an der Sozialforschungsstelle
Dortmund. Arbeitsschwerpunkte: Nationale
und internationale Arbeits- und Betriebszeit-
forschung, Work-Life-Balance, Handlungs-
theorien.
e-mail: gross@sfs-dortmund.de
Hartmut Seifert, Dr. Leiter der Abteilung WSI
in der Hans-Böckler-Stiftung. Arbeitsschwer-
punkte: Arbeitszeitpolitik und Arbeitsmarkt-
forschung.
e-mail: hartmut-seifert@boeckler.de
Georg Sieglen, Dipl. Sozialwissenschafter in
der Regionaleinheit NRW des Instituts für
Arbeitsmarkt- und Berufsforschung (IAB).
e-mail: georg.sieglen@iab.de

Hermann Groß, Dr., wissenschaftlicher Angestellter an der Sozialforschungsstelle theorien.

e-mail: gross@sfs-dortmund.de Hartmut Seifert, Dr. Leiter der Abteilung WSI in der Hans-Böckler-Stiftung. Arbeitsschwerforschung e-mail: hartmut-seifert@boeckler.de der Regionaleinheit NRW des Instituts für e-mail: georg.sieglen@iab.de 
grenzung zwischen Normalarbeitszeit und flexibler Arbeitszeit begrifflich zu klären. Sowohl Normalarbeitszeit als auch flexible Arbeitszeitmuster sind in der Literatur unterschiedlich definiert (Kurz-Scherf 1995; Herrmann et al. 1999). Für die Gestaltung der Arbeitszeit relevant erscheint uns die Definition, die die zuständigen Akteure die Tarifparteien und der Gesetzgeber - anbieten. Sie verstehen den „Normalarbeitszeitstandard" in einem operationalen Sinne und begreifen ihn als eine Referenzgröße, die den Beschäftigten basalen Schutz gewährt. Unter „Normalarbeitszeit“ wird dabei in einer engen Definition die tariflich vereinbarte Regelarbeitszeit von Vollzeitbeschäftigten verstanden, die während der Tageszeit in gleichmäßigen Proportionen an fünf Werktagen (in der Regel montags bis freitags) geleistet wird. ${ }^{1}$ In Abgrenzung hierzu gelten sämtliche abweichenden Arbeitszeitformen als flexibel. Insofern ist die Normalarbeitszeit ein wichtiger analytischer Bezugspunkt, um die vielfältigen Arbeitszeitmuster ordnen und bewerten $\mathrm{zu}$ können.

Für ein Festhalten an der normgebenden Funktion der Normalarbeitszeit sprechen aber ungeachtet ihrer schwindenden empirischen Relevanz ihre Schutzfunktionen, die sie für sowohl gesellschaftliche als auch lebensweltliche (individuelle oder familiale) Interessen und Anforderungen bieten kann. Dabei darf natürlich nicht übersehen werden, dass die Anforderungen an die Zeitgestaltung nicht statisch sind. Zu prüfen ist deshalb, ob und inwieweit angesichts sich ändernder betrieblicher Anforderungen auf der einen Seite, und angesichts sich wandelnder Arbeits- und Lebensentwürfe, Werthaltungen und Zeitpräferenzen der Beschäftigten auf der anderen Seite andere Zeitformen die Schutzfunktionen besser ausfüllen können. Unter dem Aspekt des Beschäftigtenschutzes sichert ein solcher „Normalarbeitszeitstandard“ die klare Grenzziehung von Arbeitszeit und Freizeit und damit die privatautonome Ausgestaltung lebensweltlicher (Freizeit-) Sphären. Eine neu definierte Normalarbeitszeit, die den veränderten zeitpolitischen Rahmenbedingungen Rechnung trägt, würde aber zu kurz greifen, wenn sie sich auf die Schutzfunktionen beschränken und nicht auch weitergehende Gestaltungsoptionen einschließen würde, wie sie beispielsweise das Konzept der "garantierten Optionalität" (Hinrichs 1988) vorschlägt.
Auf die Schutzfunktionen ließe sich verzichten, wenn erstens von Gleichwertigkeit unterschiedlicher Arbeitszeitformen im Hinblick auf ihren Nutzen auszugehen wäre. Und zweitens müssten die Beschäftigten ohne Einschränkungen allein nach dem Freiwilligkeitskriterium zwischen den unterschiedlichen Arbeitszeitformen wählen können. Da aber keine der beiden Voraussetzungen auch nur annäherungsweise erfüllt ist, ist die Schutzfunktion der Normalarbeitszeit weiterhin als Orientierungspunkt anzusehen. Folgende Kriterien präzisieren diese Funktion (Seifert 1995).

(1) Die Normalarbeitszeit soll die Gesundheit der Beschäftigten vor gefährdenden Belastungen infolge überlanger Arbeitszeiten schützen. Der Grad physischer und psychischer Belastungen hängt neben der Dauer auch von der Lage und der Verteilung der Arbeitszeit ab (Janßen/Nachreiner 2004). Für den Gesamtgrad der Belastungen entscheidend ist, in welchem Verhältnis Dauer, Lage und Verteilung der Arbeitszeit kombiniert werden. Die drei Dimensionen können sich in den Belastungen kumulativ verstärken und umgekehrt auch ausgleichen. So sinken mit abnehmender Dauer der Arbeitszeit die Belastungen ungünstiger Arbeitszeitlagen während der Nacht oder im Wechselschichtsystem, und natürlich gilt auch der umgekehrte Zusammenhang.

(2) Lebensweltliche und familiale Anforderungen an die Zeitgestaltung setzen nicht nur bestimmte Zeitkontingente voraus. Ebenso wichtig ist deren Verfügbarkeit zu bestimmten Zeitpunkten und -phasen. Nacht- und Wochenendarbeit, aber auch fremdbestimmte variable Arbeitszeiten können Synchronisationsprobleme verursachen.

(3) Ähnliche zeitliche Zusammenhänge gelten für die Teilhabe am sozialen, kulturellen und politischen Leben.

(4) Bei variablen Arbeitszeitformen kommt dem Autonomiegrad eine Art Schlüsselfunktion zu; er wird zur wichtigen Voraussetzung der zuvor genannten Funktionen.

(5) Schließlich garantiert die Normalarbeitszeit ein regelmäßig bezahltes und subsistenzsicherndes Einkommen und fungiert wie eine "Sperrklinke“ gegen abweichende Bestrebungen.
Die faktisch schwindende Bedeutung der Normalarbeitszeit wirft die Frage nach ihrer normativen Funktion auf. Zweifelsohne kann sich die soziale Geltung von Normen oder normativen Referenzgrößen im Zeitverlauf wandeln. Damit ist aber deren grundsätzliche Gültigkeit noch nicht zur Disposition gestellt. Normen oder normativen Referenzgrößen muss eine raum- und zeitunabhängige Gültigkeit zukommen, weil diese andernfalls ihre regulierenden und die abhängig Beschäftigten schützenden Funktionen nicht erfüllen könnten. Dies gilt insbesondere für die oben skizzierten Schutzfunktionen der ,Normalarbeitszeit“. Allerdings besteht zwischen sozialer Geltung und „raum- und zeitloser“ Gültigkeit von Normen oder Standards eine interne Beziehung. Diese lässt sich mit Bezug auf den oben angeführten Befund so umschreiben, dass die Erosion der sozialen Geltung von Normen oder Standards nicht so weit gehen darf, dass dadurch deren Gültigkeit folgenreich entwertet wird.

\section{Fragestellungen und Hypothesen}

Angesichts der abnehmenden sozialen Gültigkeit der Normalarbeitszeit gehen die nachfolgenden Analysen der Frage nach, welcher Typ von Beschäftigten noch unter Bedingungen der Normalarbeitszeit tätig ist und welche soziodemografischen, qualifikations-, tätigkeits-, einkommens- und familienstandsbezogenen Merkmale diese Beschäftigten aufweisen. Werden diese Tätigkeitsstrukturen die zukünftige Erwerbsarbeit prägen oder handelt es sich eher um „Auslaufmodelle“, sodass damit zu rechnen ist, dass die Normalarbeitszeit weiter an Bedeutung verlieren wird?

\footnotetext{
Sicherlich handelt es sich hierbei um eine idealtypische Form der Normalarbeitszeit. Abweichun gen gehörten stets zur Normalität der Arbeitszeitgestaltung. So war auch nach Einführung der 5-Tagewoche der Samstag für die Beschäftigten im Einzelhandel oder in Teilen des Transportwesens, des Hotel- und Gaststättengewerbes oder der Sicherheitsdienste ein regelmäßiger Arbeitstag, wenngleich jeweils nur ein Teil der Beschäftigten an diesem Tag arbeiten musste. Die Arbeitszeit an diesem Tag wurde auch nicht geson dert vergütet.
} 
Unsere These ist, dass abhängig Beschäftigte heute eher „multiplen“ Anforderungen der Arbeitszeitflexibilisierung ausgesetzt sind. Anders formuliert: Die nach den Dimensionen der Arbeitszeit unterschiedenen Formen der Arbeitszeitflexibilisierung schließen sich nicht wechselseitig aus, sondern können sich eher kumulativ überlagern und verstärken. Diese These wird durch den Befund unterstützt, dass heute fast jeder zweite Beschäftigte in den „hoch- oder multiflexiblen“ Arbeitszeitkontenmodellen tätig ist (Groß/Schwarz 2006). Angesichts der komplexer gewordenen Arbeitszeitmuster gilt es in den Analysen insbesondere zu überprüfen, in welchem Ausmaß sich die extremen Formen der Arbeitszeitflexibilisierung voneinander unterscheiden oder miteinander kombiniert werden. Schließen sich einige Formen eher aus oder treten sie kumulativ auf?

Diese Fragen wurden bislang theoretisch nicht modelliert. Da auch nur wenige ökonomische Hypothesen formuliert werden können, geht es primär darum, die Merkmale zu identifizieren, die Beschäftigte mit extremen Arbeitszeiten charakterisieren. Betrachtet man die möglichen erklärenden Variablen, dann dürfte die Betriebsgröße für die einzelnen Formen der Arbeitszeit eine jeweils unterschiedliche Bedeutung haben. Extreme Schichtformen dürften in größeren Betrieben häufiger anzutreffen sein, da stoffumwandelnde Produktionsprozesse (Stahl-, Glas-, chemische Industrie) mit zumindest in Teilbereichen kapitalintensiven Anlagen bestimmte betriebliche Mindestgrößen voraussetzen. Ebenso erfordern bestimmte Dienstleistungstätigkeiten (Hotel- und Gastgewerbe, Pflege- und Gesundheitsdienste) oft einen Rund-um-die-Uhr-Service. Diese Bereiche sind eher klein- und mittelbetrieblich organisiert. Im Hinblick auf die Dauer dürften überlange Arbeitszeiten eher in Kleinbetrieben vorkommen, da hier nur selten Betriebsratsgremien existieren, die eine kontrollierende und begrenzende Funktion ausüben (vgl. dazu den Beitrag von Ellguth/Promberger in diesem Heft). Extreme Schichtarbeit schließt aus zeitorganisatorischen aber auch aus Belastungsgründen überlange Arbeitszeiten ebenso aus wie stark variabilisierte Arbeitszeiten.

In einem engen Kontext mit der Betriebsgröße dürfte die Wirtschaftsstruktur als Einflussfaktor zu sehen sein. Wirtschaftsbereiche mit kapitalintensiver Produktion oder Rund-um-die-Uhr-Service- angebot sind zwangsläufig auf Mehrschichtsysteme angewiesen. Wirtschaftsbereiche, in denen Dienstleistungen nach dem Uno-actu-Prinzip erbracht werden, aber auch Produktionsbereiche mit schwankender Nachfrage werden stark variabilisierte Arbeitszeiten einsetzen. Mit Hilfe solcher flexiblen Arbeitszeitmuster lassen sich Lagerkosten, Überstundenzuschläge und Leerzeiten reduzieren.

In die Schätzungen einbezogen wird ferner das Alter der Beschäftigten. Die Wahrscheinlichkeit, dass jüngere Beschäftigte seltener in Normalarbeitszeit arbeiten, könnte damit zu tun haben, dass bei ihnen die Bereitschaft zu überlangen Arbeitszeiten aus Einkommens- und Karrieregründen ausgeprägter ist als bei anderen Altersgruppen. Sie sind vermutlich zudem eher bereit und in der Lage, extreme Schichtarbeit zu leisten oder stark schwankende Arbeitszeiten zu akzeptieren. Gegen diese Annahme könnte sprechen, dass in der Familiengründungsphase außerbetriebliche Zeitbedarfe die Wahrscheinlichkeit der Normalarbeitszeit erhöhen. Als Kontrollvariable wird deshalb der familiale Status einbezogen. Zusätzlich könnte das Geschlecht der Beschäftigten bedeutsam für die Verteilung von extremen Formen flexibler Arbeitszeiten sein. Frauen dürften aufgrund der nach wie vor noch verbreitet existierenden Doppelbelastungen durch Familie und Beruf weniger in der Lage sein, überlange Arbeitszeiten im Rahmen ausgeprägter Überstundenarbeit zu leisten (Schank/Schnabel 2004). Weniger eindeutige Annahmen lassen sich über das Auftreten extremer Schichtarbeit machen. Frauen sind einerseits in den traditionellen Industriesektoren mit hoher Kapitalintensität unterrepräsentiert (Jirjahn 2004). Zudem könnte hier das bis 1992 geltende Nachtarbeitsverbot für Arbeiterinnen nachwirken. Andererseits ist der Frauenanteil in Teilbereichen des Dienstleistungssektors mit einem zeitlich durchgehenden Serviceangebot hoch.

Auch der betriebliche Status dürfte das Arbeitszeitmuster wesentlich beeinflussen. Beschäftigte in Leitungsfunktionen üben längere Arbeitszeiten aus, verfügen über größeren Spielraum bei der Gestaltung bzw. der Verteilung der Arbeitszeit und sind dafür aber seltener in extreme Schichtsysteme eingebunden als Beschäftigte mit geringen oder keinen Führungsaufgaben. Lange Arbeitszeiten gelten bei Führungskräften als Äquivalent für außertarifliche
Entlohnung. Der gleichzeitig hohe Grad an selbstbestimmter Arbeitsorganisation erlaubt zugleich ein hohes Maß an zeitlicher Souveränität.

\section{Methodischer Ansatz}

Ziel der nachfolgenden Studie ist es, die prägenden Faktoren für die verschiedenen Formen von Arbeitszeitflexibilisierung herauszuarbeiten. Die empirische Grundlage der bi- und multivariaten Analysen bilden die Daten der repräsentativen Beschäftigtenbefragung des ISO-Instituts „Arbeitszeit 2003“ (Bauer et al. 2004). Mit den Daten der ISO-Arbeitszeiterhebung von 2003 kann erstmals untersucht werden, welche extremen Formen der Arbeitszeitflexibilisierung - in Relation zur Normalarbeitszeit - bei welchen Beschäftigten wahrscheinlich anzutreffen sind.

Die vielfältigen, empirisch belegten Formen von Arbeitszeitflexibilisierung erfordern ein stimmiges Analyseschema, mit dem sich das Dickicht der praktizierten Arbeitszeitflexibilisierung aufhellen lässt. Um die Komplexität zu reduzieren, werden in einem ersten Schritt die verschiedenen Formen der Arbeitszeitflexibilisierung nach den drei Dimensionen von Arbeitszeit (Dauer, Lage, Verteilung) unterschieden. Der zweite Schritt soll die (möglichen) Kontraste weiter schärfen. Deshalb bilden wir Kontrastgruppen von extremen Formen der Arbeitszeitflexibilisierung. Deren Konstruktion erfolgt entlang der Dimensionen Dauer, Lage und Verteilung der Arbeitszeit. Dementsprechend betrachten wir Gruppen von Beschäftigten

- mit überlangen tatsächlichen Wochenarbeitszeiten von 42 und mehr Stunden pro Woche;

- mit extremen Schwankungen der wöchentlichen Arbeitszeit von 20 und mehr Stunden;

- in extremen Formen von Schicht- und Nachtarbeit (in Schicht-Systemen, die 24 Stunden am Tag an fünf, sechs oder sieben Tagen in der Woche „laufen“, wodurch die Arbeitszeitlage der Beschäftigten hinsichtlich des Wochentags - montags bis sonntags - und/oder der täglichen Anfangszeiten - früh, spät und/oder nachts - stark variiert). 


\begin{tabular}{|c|c|c|c|c|c|}
\hline & $\begin{array}{l}\text { Beschäftigte } \\
\text { in Normal- } \\
\text { arbeitszeit }\end{array}$ & $\begin{array}{c}\text { Beschäftigte } \\
\text { mit überlangen } \\
\text { Wochenarbeits- } \\
\text { zeiten }\end{array}$ & $\begin{array}{l}\text { Beschäftigte } \\
\text { mit extrem } \\
\text { schwankenden } \\
\text { Arbeitszeiten }\end{array}$ & $\begin{array}{l}\text { Beschäftigte } \\
\text { in kontinuier- } \\
\text { licher oder teil- } \\
\text { kontinuierlicher } \\
\text { Schicht- und } \\
\text { Nachtarbeit }\end{array}$ & Insgesamt \\
\hline \multicolumn{6}{|l|}{ Wirtschaftszweig } \\
\hline Primärer Sektor & 4,7 & 4,0 & 5,2 & 2,4 & 3,6 \\
\hline Sekundärer Sektor & 22,5 & 24,3 & 18,0 & 50,2 & 23,6 \\
\hline Bau & 11,3 & 10,9 & 9,8 & - & 6,6 \\
\hline $\begin{array}{l}\text { Distributive } \\
\text { Dienstleistungen }\end{array}$ & 14,0 & 15,7 & 11,4 & 9,5 & 15,3 \\
\hline $\begin{array}{l}\text { Unternehmensbezogene } \\
\text { Dienstleistungen }\end{array}$ & 10,2 & 13,7 & 15,7 & 2,4 & 10,8 \\
\hline Soziale Dienstleistungen & 29,1 & 26,2 & 30,7 & 31,3 & 31,2 \\
\hline $\begin{array}{l}\text { Persönliche } \\
\text { Dienstleistungen }\end{array}$ & 8,3 & 5,3 & 8,2 & 4,3 & 8,5 \\
\hline \multicolumn{6}{|l|}{ Betriebsgrößenklassen } \\
\hline 1-4 Beschäftigte & 10,2 & 8,3 & 7,4 & 1,8 & 11,3 \\
\hline 5-99 Beschäftigte & 68,0 & 54,6 & 52,8 & 25,5 & 55,1 \\
\hline $100-499$ & 14,1 & 21,3 & 24,0 & 34,0 & 18,9 \\
\hline 500 u. m. Beschäftigte & 7,7 & 15,8 & 15,8 & 39,2 & 14,7 \\
\hline \multicolumn{6}{|l|}{ Geschlecht } \\
\hline männlich & 57,7 & 73,9 & 69,1 & 71,6 & 52,9 \\
\hline weiblich & 42,3 & 26,1 & 30,9 & 28,4 & 47,1 \\
\hline \multicolumn{6}{|l|}{ Alter } \\
\hline $18-25$ & 14,5 & 9,4 & 8,6 & 7,7 & 10,1 \\
\hline $26-40$ & 40,7 & 41,0 & 43,9 & 49,1 & 40,8 \\
\hline $41-60$ & 41,7 & 46,6 & 44,1 & 40,1 & 45,8 \\
\hline $61-65$ & 3,0 & 3,0 & 3,4 & 3,2 & 3,4 \\
\hline \multicolumn{6}{|l|}{ Haushaltsstand } \\
\hline Single & 39,6 & 31,0 & 29,0 & 24,8 & 30,0 \\
\hline Alleinerziehend & 4,5 & 2,5 & 3,3 & 1,9 & 5,1 \\
\hline Paar ohne Kind & 31,0 & 32,9 & 32,5 & 34,3 & 32,6 \\
\hline Paar mit Kind & 24,9 & 33,7 & 35,3 & 39,0 & 32,4 \\
\hline \multicolumn{6}{|l|}{ Betrieblicher Status } \\
\hline Hoch & 11,9 & 36,5 & 39,8 & 18,1 & 21,5 \\
\hline Mittel & 59,6 & 46,8 & 42,5 & 57,0 & 51,4 \\
\hline Niedrig & 28,5 & 16,7 & 17,7 & 24,9 & 27,0 \\
\hline \multicolumn{6}{|c|}{ Persönliches Nettoeinkommen } \\
\hline Bis $999 €$ & 13,1 & 7,8 & 15,1 & 7,0 & 24,5 \\
\hline $1000-1999 €$ & 77,3 & 56,9 & 55,8 & 65,2 & 55,5 \\
\hline 2000 u. m. $€$ & 9,6 & 35,3 & 29,1 & 27,8 & 20,0 \\
\hline Insgesamt & 12,8 & 28,9 & 15,2 & 5,6 & 100,0 \\
\hline
\end{tabular}

Diese Kontrastgruppen vergleichen wir mit der Gruppe der Beschäftigten, die unter den Bedingungen von Normalarbeitszeit tätig sind, anhand identischer Merkmale, um die Unterschiede zwischen diesen Gruppen so scharf wie möglich herauszuarbeiten. Die Normalarbeitszeit ist definiert als eine Vollzeitbeschäftigung zwischen 35 und 42 Stunden vertraglicher und auch faktischer Arbeitszeit, die sich auf fünf Wochentage - montags bis freitags - verteilt und in der Lage nicht variiert, das heißt, Überstundenarbeit, Schicht-, Nachtund Wochenendarbeit, Arbeit mit schwankender Arbeitszeitverteilung und Gleitzeitarbeit ausschließt. Die Konzentration auf Beschäftigtengruppen mit extremen Ar-

nurde untersucht, welchen Einfluss Tätigkeitsstrukturen, betriebliche, soziodemografische, familienstands-, qualifikations- und einkommensbezogene Merkmale auf die genannten Formen der Arbeitszeit ausüben: auf die Normalarbeitszeit, auf überdurchschnittlich lange Arbeitszeiten, auf extrem variable Arbeitszeiten und auf Arbeitszeiten mit kon- tinuierlichen oder teilkontinuierlichen Schichtsystemen. Dazu wurden binäre logistische Regressionen für diese vier dummy-codierten abhängigen Variablen durchgeführt (Hosmer/Lemeshow 1989; Backhaus et al. 2003). Auf Basis des oben genannten Datensatzes erfolgten die Modellschätzungen iterativ, das heißt in drei Schritten, wobei die Analyse um jeweils einen Block von erklärenden Variablen erweitert wurde. Zunächst wurde die Analyse nur mit den Variablen der unterschiedlichen Tätigkeitsstrukturen durchgeführt; dann wurde diese Analyse mit einigen Merkmalen der Betriebe, in denen die Befragten tätig sind, ergänzt; schließlich wurden noch soziodemografische, qualifikations-, familienstands- und einkommensbezogene Merkmale der Beschäftigten in die Analyse einbezogen. Bei den hinzugenommenen Kontrollvariablen handelt es sich um nominal- bzw. ordinalskalierte Variablen, für die als Kategorial-Codierung der Abweichungskontrast gewählt wurde. Aus Darstellungsgründen enthält Tabelle 2, in der die Regressionsgleichungen aufgeführt werden, nur die Beta-Koeffizienten.

\section{Ergebnisse}

\section{1 ÜBERSICHT}

Die Untersuchungsgruppen mit extremen Formen atypischer bzw. flexibler Arbeitszeit repräsentieren insgesamt 39,6\% der abhängig Beschäftigten (Tabelle 1). ${ }^{2}$ Nur noch jede bzw. jeder achte Beschäftigte arbeitet auf Basis der hier definierten Normalarbeitszeit. Gegenüber 1989 hat sich der Anteil der Beschäftigten mit Normalarbeitszeit halbiert (Groß et al. 1989). Diese Arbeitszeitform ist mit über drei Viertel der Beschäftigten $(78,2 \% \mathrm{zu} 66,4 \% \mathrm{im}$ Durchschnitt) überdurchschnittlich häufig in den Kleinst- und Kleinbetrieben (1-99 Beschäftigte) zu finden. Bei den Beschäftigten handelt es sich überdurchschnittlich häufig um „Singles“ (39,6 \% zu 30,0 \%)
2 In der Analyse ausgeblendet bleiben vor allem Beschäftigte mit Arbeitszeiten von weniger als 35 Stunden und moderate Formen der Arbeitszeitflexibilisierung, wie z. B. Zweischichtarbeit. Die hier gebildeten Arbeitszeitformen sind nicht ganz trennscharf und überlappen sich teilweise. 
mit eher mittlerem oder niedrigem betrieblichen Status $(88,1 \% \mathrm{zu} 78,4 \%)$. Demgegenüber sind Beschäftigte in extremer Schichtarbeit überwiegend männlich (71,6 \% zu 52,9 \%) und überdurchschnittlich häufig in Mittel- und Großbetrieben (100 und mehr Beschäftigte) tätig (73,2 \% zu 33,6 \%) - vornehmlich im Sekundären Sektor (50,2 \% zu 23,6 \%). Ein nicht unerheblicher Teil der Beschäftigten (28,9\%) leistet überlange Wochenarbeitszeiten, überdurchschnittlich häufig bei den unternehmensbezogenen Dienstleistungen $(13,7 \%$ zu 10,8 \%) und im Baugewerbe $(10,9 \% \mathrm{zu} 6,6 \%)$. Auch hier dominieren die männlichen Beschäftigten $(73,9 \% \mathrm{zu}$ $52,9 \%$ ). Beschäftigte mit extrem schwankenden täglichen oder wöchentlichen Arbeitszeiten sind überdurchschnittlich häufig im Bereich der unternehmensbezogenen Dienstleistungen (15,7 \% zu 10,8 \%) und in Mittel- und Großbetrieben (39,8\% zu 33,6\%) tätig.

\section{2 MULTIVARIATE ANALYSEN}

\section{NORMALARBEITSZEIT}

Die Normalarbeitszeit ist, wie die Koeffizienten in Tabelle 2 zeigen, am ehesten bei Beschäftigten in Klein- und Mittelbetrieben (5-99 Beschäftigte) mit einem durchschnittlichen Nettoeinkommen zwischen 1.000 und $1.999 €$ zu erwarten. Es handelt sich im Wesentlichen um Beschäftigte mit einer relativ langen Betriebszugehörigkeitsdauer und in mittlerer oder niedriger beruflicher Position. Mit der Betriebsgröße dürfte zusammenhängen, dass die Existenz eines Betriebsrates für das Arbeiten unter Bedingungen der Normalarbeitszeit nicht von ausschlaggebender Bedeutung ist; denn Betriebsräte sind in Betrieben mit weniger als 100 Beschäftigten deutlich seltener als in Großbetrieben anzutreffen. Soziodemografische Merkmale spielen für das „Arbeiten unter Bedingungen der Normalarbeitszeit" keine prägende Rolle.

Für die Normalarbeitszeit charakteristisch sind Tätigkeitsstrukturen mit einem hohen Grad an Standardisierung und Detailkontrolle. Hauptsächlich dürfte es sich um eine tayloristische und stark routinisierte Arbeitsorganisation handeln, die kaum innovatives Arbeitshandeln erfordert. Bestimmend sind vielmehr enge Sollvorgaben für Zeitpunkt und Tempo der Arbeit. Dem entspricht, dass diese Tätigkeiten durch einen engen zeitlichen Gestaltungs- spielraum und nur geringen Abstimmungsbedarf mit den Kollegen gekennzeichnet sind.

\section{ÜBERLANGE ARBEITSZEITEN}

Für die Analyse der Auftretenswahrscheinlichkeit von tatsächlichen Arbeitszeiten über 42 Stunden pro Woche wurden - ergänzend zu den bisher berücksichtigten unabhängigen Variablen - zusätzlich die Verteilung und Steuerung der täglichen Arbeitszeiten berücksichtigt. ${ }^{3}$ In dieser Gruppe sind eher alleinstehende, männliche Beschäftigte mit hohem betrieblichen Status und einem überdurchschnittlichen Nettoeinkommen anzutreffen. Die Betriebszugehörigkeitsdauer ist vergleichsweise kurz. Bei den Branchen stechen das Baugewerbe und mit Abstrichen der Bereich der distributiven Dienstleistungen hervor, wohingegen die Betriebsgröße keine Rolle spielt. Mit hohem beruflichem Status und überdurchschnittlichem Nettoeinkommen korrespondiert, dass diese Gruppe geradezu kontrastierend zur vorgenannten Gruppe Anfangs- und Endzeiten der täglichen Arbeitszeit weitgehend selbst steuert und sich von daher auch stärker mit anderen Beschäftigten bei der Ausführung der jeweiligen Tätigkeiten abstimmen muss. Das auf eine tayloristische Arbeitsorganisation verweisende Merkmal der Detailkontrolle hat keine prägende Bedeutung. Dagegen deutet der hohe Beta-Wert der selbst gesteuerten Variation der täglichen Arbeitszeit auf deren Zusammenhang mit überlangen Wochenarbeitszeiten hin (Tabelle 2).

\section{EXTREME SCHWANKUNGEN DER ARBEITSZEIT}

Die Beschäftigtengruppe mit extremen wöchentlichen Schwankungen der Arbeitszeit gleicht in der Prägekraft einer Reihe von Merkmalen (männliches Geschlecht, hoher betrieblicher Status, selbst gesteuerte Variation der täglichen Arbeitszeiten) der Gruppe mit überlangen Arbeitszeiten und kontrastiert mit der Gruppe der Beschäftigten, die unter Bedingungen des Normalarbeitszeitstandards tätig sind. Am deutlichsten zeigt sich dies beim Merkmal der tatsächlichen Arbeitszeit von 45 und mehr Stunden pro Woche. Der hohe BetaKoeffizient bedeutet, dass mit der Dauer der tatsächlichen Arbeitszeit die Wahrscheinlichkeit extrem schwankender Arbeitszeiten steigt.
Die Dauer der Betriebszugehörigkeit indiziert kurzfristige und eher relativ instabile Beschäftigungskarrieren. In Betrieben mit Betriebsrat ist eher mit extremen Schwankungen der Arbeitszeit zu rechnen als in Betrieben ohne. Dies dürfte damit zusammenhängen, dass extreme Schwankungen der Arbeitszeit Regelungen erfordern. Sieht man einmal von dem Fall der Selbstregulierung durch die sogenannte Vertrauensarbeitszeit ab, werden Schwankungen der Arbeitszeit normalerweise durch Arbeitszeitkonten reguliert. Deren Funktionstüchtigkeit erfordert von den Betriebsparteien den Abschluss von formalisierten Vereinbarungen (oder auch von informellen Übereinkünften), in denen wenigstens rudimentär neben den Interessen des Betriebes auch die der Beschäftigten berücksichtigt werden.

Stark variierende Arbeitszeiten leisten eher Beschäftigte im mittleren Alter und in einem Paarhaushalt mit Kind als in anderen Alters- oder Haushaltsgruppen. Dieser Befund deutet darauf hin, dass für einen nicht nur marginalen Teil von Beschäftigten die Phasen der Konsolidierung im Beruf, wofür bisweilen extreme Schwankungen der Arbeitszeit in Kauf genommen werden, und der Gründung einer Familie zusammenfallen. Für diesen Teil der Beschäftigten dürfte sich deshalb Vereinbarkeit von Beruf und Familie kaum erzielen lassen, wenn sie vornehmlich über einen Zugewinn von erwerbsarbeitsfreier Zeit angestrebt wird. Sie muss ebenso innerhalb der doppelten Anforderungen von beruflicher Konsolidierung und Familiengründung gesucht werden.

\section{EXTREME SCHICHTARBEIT}

Flexibilisierung aufgrund von extremer Schichtarbeit grenzt sich deutlich ab von den Flexibilisierungen der Arbeitszeitverteilung und der Arbeitszeitdauer. Extreme Schichtarbeit ist, was die Tätigkeitsstrukturen anbelangt, vor allem in Bereichen mit tayloristischer Arbeitsorganisation (Detail-

\footnotetext{
3 Die Ergebnisse aus "Arbeitszeit 2003“ (Bauer et a 2004, S. 102f.) zeigen, dass insbesondere bei Beschäftigten mit der Möglichkeit, über die täglichen Anfangs- und Endzeiten selbst zu entscheiden, die tatsächlichen Arbeitszeiten deutlich häufiger über den vertraglichen Arbeitszeiten liegen als bei Beschäftigten mit zwar variierenden, aber überwiegend betrieblich gesteuerten Arbeitszeiten oder mit starren Anfangs- und Endzeiten.
} 
Tabelle 2: Logistische Regressionen (Regressionskoeffizient Beta)

\begin{tabular}{|c|c|c|c|c|}
\hline & $\begin{array}{l}\text { Normal- } \\
\text { arbeitszeit }\end{array}$ & $\begin{array}{c}\text { Überlange } \\
\text { Wochen- } \\
\text { arbeitszeiten }\end{array}$ & $\begin{array}{c}\text { Extrem } \\
\text { schwankende } \\
\text { Arbeitszeiten }\end{array}$ & $\begin{array}{l}\text { Kontinuierliche } \\
\text { oder teil- } \\
\text { kontinuierliche } \\
\text { Schichtarbeit }\end{array}$ \\
\hline \multicolumn{5}{|l|}{$\begin{array}{l}\text { Betriebliche Merkmale } \\
\text { Tätigkeitsprofile }\end{array}$} \\
\hline Detailkontrolle & $0,211 * * *$ & $-0,198^{* * *}$ & $-0,051$ & $0,446 * * *$ \\
\hline Abstimmungsbedarf & $-0,113+$ & $0,144^{* *}$ & $0,123+$ & $0,411 * * *$ \\
\hline Zeitlicher Gestaltungsspielraum & 0,022 & $-0,166^{* *}$ & $-0,060$ & $-0,786 * * *$ \\
\hline \multicolumn{5}{|l|}{ Branche } \\
\hline Primärer Sektor & $\mathrm{R}$ & $\mathrm{R}$ & $\mathrm{R}$ & $\mathrm{R}$ \\
\hline Sekundärer Sektor & $-0,418$ & $-0,084$ & $-1,046^{* *}$ & $0,245^{*}$ \\
\hline Bau & $-0,025$ & $0,574 *$ & 0,130 & $-7,219$ \\
\hline Distributive DL & $-0,479$ & 0,217 & $-0,649 *$ & $-0,440$ \\
\hline Unternehmensb. DL & $-0,197$ & 0,049 & $-0,200$ & $-1,643 *$ \\
\hline Personenb. DL & $-0,348$ & 0,008 & 0,271 & $-0,159$ \\
\hline Soziale DL & $-0,229$ & $-0,284$ & $-0,001$ & $0,203 *$ \\
\hline \multicolumn{5}{|l|}{ Betriebsgröße } \\
\hline 1-4 Besch. & $\mathrm{R}$ & $\mathrm{R}$ & $\mathrm{R}$ & $\mathrm{R}$ \\
\hline 5-99 Besch. & $0,003^{*}$ & 0,050 & $-0,170$ & 0,793 \\
\hline 100-499 Besch. & $-0,732 * *$ & $-0,053$ & $-0,293$ & $1,795^{*}$ \\
\hline $500+$ Besch. & $-0,833 * *$ & $-0,150$ & $-0,341$ & $2,072 * *$ \\
\hline Betriebsrat & $-0,271+$ & $-0,151$ & $0,347^{*}$ & $1,085 * *$ \\
\hline \multicolumn{5}{|l|}{ Betrieblicher Status } \\
\hline Hoch & $-0,523+$ & $0,491 * *$ & $0,836 * * *$ & $-0,191$ \\
\hline Mittel & $-0,026$ & $-0,052$ & 0,170 & $-0,050$ \\
\hline Niedrig & $\mathrm{R}$ & $\mathrm{R}$ & $\mathrm{R}$ & $\mathrm{R}$ \\
\hline \multicolumn{5}{|l|}{ Betriebszugehörigkeit } \\
\hline 10 Jahre und mehr & $0,324+$ & $-0,383^{* *}$ & $-0,411^{*}$ & 0,362 \\
\hline 3-9 Jahre & 0,082 & $-0,231+$ & $-0,355^{*}$ & $0,723 *$ \\
\hline Bis 2 Jahre & $\mathrm{R}$ & $\mathrm{R}$ & $\mathrm{R}$ & $\mathrm{R}$ \\
\hline \multicolumn{5}{|l|}{ Soziodemographische Merkmale } \\
\hline \multicolumn{5}{|l|}{ Geschlecht } \\
\hline männlich & 0,195 & $0,787^{* * *}$ & $0,423^{*}$ & 0,332 \\
\hline \multicolumn{5}{|l|}{ Alter } \\
\hline 61-65 Jahre & $-0,136$ & $-0,504$ & $-0,180$ & 0,444 \\
\hline 41-60 Jahre & $-0,395+$ & $-0,090$ & 0,096 & $-0,034$ \\
\hline 26-40 Jahre & $-0,253$ & $-0,056$ & $0,130^{*}$ & 0,324 \\
\hline 18-25 Jahre & $\mathrm{R}$ & $\mathrm{R}$ & $\mathrm{R}$ & $\mathrm{R}$ \\
\hline \multicolumn{5}{|l|}{ Familienstand } \\
\hline Single & 0,114 & $0,538^{*}$ & 0,342 & $-0,366$ \\
\hline Alleinerziehend & $\mathrm{R}$ & $\mathrm{R}$ & $\mathrm{R}$ & $\mathrm{R}$ \\
\hline Paare ohne Kind & $-0,084$ & 0,339 & 0,267 & 0,401 \\
\hline Paare mit Kind & $-0,199$ & 0,314 & $0,464^{*}$ & $0,426^{*}$ \\
\hline \multicolumn{5}{|l|}{ Persönliches Nettoeinkommen } \\
\hline $2000+€$ & $0,528^{*}$ & $1,727^{* * *}$ & 0,154 & $1,132 *$ \\
\hline $1000-1999 €$ & $1,193 * * *$ & $1,175^{* * *}$ & $0,404+$ & $0,792+$ \\
\hline Bis $999 €$ & $\mathrm{R}$ & $\mathrm{R}$ & $\mathrm{R}$ & $\mathrm{R}$ \\
\hline \multicolumn{5}{|l|}{$\begin{array}{l}\text { Merkmale der Arbeitszeit- } \\
\text { gestaltung }\end{array}$} \\
\hline \multicolumn{5}{|l|}{ Arbeitszeitsteuerung } \\
\hline $\begin{array}{l}\text { Selbstgesteuerte Variation der } \\
\text { Arbeitszeiten }\end{array}$ & & $0,886^{* * *}$ & $1,156 * * *$ & \\
\hline $\begin{array}{l}\text { Betrieblich gesteuerte Variation } \\
\text { der Arbeitszeiten }\end{array}$ & & $0,290^{*}$ & $1,186 * * *$ & \\
\hline Keine Variation & & $\mathrm{R}$ & $\mathrm{R}$ & \\
\hline \multicolumn{5}{|l|}{ Tatsächliche Wochenarbeitszeit } \\
\hline 1-34 Stunden & & & $\mathrm{R}$ & $\mathrm{R}$ \\
\hline 35-40 Stunden & & & $-0,637^{* *}$ & $0,565^{*}$ \\
\hline 41-44 Stunden & & & 0,249 & 0,201 \\
\hline $45+$ Stunden & & & $1,159 * * *$ & 0,542 \\
\hline Pseudo-R Quadrat & 0,117 & 0,247 & 0,264 & 0,346 \\
\hline \multicolumn{3}{|c|}{$\begin{array}{l}+P<0,1,{ }^{*} P<0,05,{ }^{*} \mathrm{P}<0,01,{ }^{* *}{ }^{*} \mathrm{P}<0,001 ; \mathrm{R}=\text { Referenzkategorie. } \\
\text { Quelle: Arbeitszeit 2003; Berechnungen der Autoren. }\end{array}$} & 1 & $\begin{array}{l}\text { Hans Böckler } \\
\text { Stiftung }\end{array}$ \\
\hline
\end{tabular}

kontrolle) zu erwarten. Im Unterschied zur Beschäftigung unter Bedingungen des Normalarbeitszeitstandards ist extreme Schichtarbeit ferner sehr wahrscheinlich in Bereichen, in denen die Arbeit mit anderen Kollegen abgestimmt werden muss: Die Mehrfachbesetzung eines Arbeitsplatzes ist auf seine „ordentliche“ Übergabe von einem Kollegen auf den nächsten zwingend angewiesen. Extreme Schichtarbeit ist weitaus mehr als alle anderen Untersuchungsvariablen stark durch Betriebsgrößenklassenmerkmale geprägt. Bei den Branchen dominieren das Verarbeitende Gewerbe und der Bereich der sozialen Dienstleistungen. Die Dauer der Betriebszugehörigkeit verweist im Kontrast zu den beiden vorgenannten Untersuchungsgruppen auf relativ stabile Beschäftigungskarrieren.

Wenig überraschend korrespondiert damit der starke Einfluss von größeren Betrieben (100 und mehr Beschäftigte). In Betrieben mit 500 und mehr Beschäftigten ist extreme Schichtarbeit wahrscheinlicher als in Bezug auf die Referenzkategorie. Damit wiederum hängt zusammen, dass in Betrieben mit betrieblicher Interessenvertretung eher mit extremer Schichtarbeit zu rechnen ist als in Betrieben ohne. Anders als bei den beiden vorgenannten Untersuchungsvariablen spielt das Merkmal Geschlecht keine differenzierende Rolle. Schichtarbeit wird von Männern (vornehmlich im Sekundären Sektor) und Frauen (vornehmlich im Bereich der sozialen Dienstleistungen) gleichermaßen geleistet.

Die soziodemografischen Merkmale haben kaum Einfluss auf die Wahrscheinlichkeit von extremer Schichtarbeit. Die Variablen Alter, Haushaltsstand und Betriebszugehörigkeit deuten allerdings darauf hin, dass extreme Schichtarbeit bei jüngeren (25 Jahre und jünger), alleinstehenden Beschäftigten mit einer kurzen Betriebszugehörigkeit (2 Jahre und weniger) eher unwahrscheinlich ist. Zudem liefert der relativ hohe Beta-Koeffizient bei Beschäftigten in Paarhaushalten mit Kindern Anhaltspunkte dafür, dass die Vereinbarkeit von Beruf und Familie wesentlich durch die Doppelbelastung aus beruflicher Konsolidierung und Familienarbeit erschwert wird und dementsprechend diskutiert werden muss.

Extreme Schichtarbeit ist (wegen der Zuschläge) mit einem überdurchschnittlichen Nettoeinkommen verbunden. Die Arbeitszeitform ist bei einer Arbeitszeit von 
35-40 Wochenstunden eher zu erwarten als bei einer Arbeitszeit von 41-45 Wochenstunden (und selbstverständlich auch bei einer Teilzeitbeschäftigung mit 1-34 Wochenstunden). Extreme Schichtarbeit hat mithin eine größere Nähe zu der Gruppe der Beschäftigten in Normalarbeitszeit als zu den beiden anderen Extremgruppen der Arbeitszeit.

\section{Schlussfolgerungen}

Die Befunde zur Normalarbeitszeit sind desillusionierend. Dieses Modell trifft faktisch nur noch für einen abnehmenden, ohnehin schon sehr geringen Teil von Beschäftigten in einem eingeschränkten Segment von Erwerbsarbeit zu. Dieses ist durch eine tayloristische Arbeitsorganisation mit engen Sollvorgaben für Zeitpunkt und Tempo der Arbeit charakterisiert. So gesehen tangiert der empirisch feststellbare Prozess, dass die soziale Geltung der Normalarbeitszeit entwertet wird, auch deren normgebende Kraft. Die Normalarbeitszeit scheint in der oben angeführten operationalen, für die relevanten Akteure immer noch verbindlichen Definition zunehmend weniger geeignet, die basalen Schutzfunktionen für die Beschäftigten erfüllen zu können. Eine Reformulierung der Normalarbeitszeit, die sich stärker an den praktizierten Formen der Arbeitszeitgestaltung orientiert, ohne dabei ihre traditionellen Schutzfunktionen aufzugeben, ist insofern bei der Neuausrichtung staatlicher und tariflicher Arbeitszeitpolitik überfällig (vgl. den Beitrag von Haipter/Lehndorff in diesem Heft).

Dabei können, wie die Analysen zeigen, überlange Wochenarbeitszeiten ebenso wenig ignoriert werden wie extrem schwankende Arbeitszeiten. Solche Arbeitszeitformen praktizieren überwiegend hoch qualifizierte Beschäftigte mit hohem Dis- positionsspielraum in der Steuerung der Arbeitszeitgestaltung in sogenannten „Zukunftsbranchen“. Von diesen Beschäftigtengruppen dürfte eine nicht unerhebliche Ausstrahlung mit Nachahmereffekten ausgehen, die die Polarisierung der Arbeitszeitgestaltung weiter verschärfen würde. Zugleich haben sich mit den Kontenmodellen Arbeitszeitmuster etabliert, die es erlauben, innerhalb eines definierten Zeitraums sowohl überlange Wochenarbeitszeiten als auch extrem schwankende Arbeitszeiten zu praktizieren, ohne dass dadurch die vertraglich vereinbarte oder tarifliche Wochenarbeitszeit außer Kraft gesetzt wird. Arbeitszeitkonten haben sowohl einen deregulierenden als auch reregulierenden Effekt und scheinen von daher geeignet, eine Vorlage für die Reformulierung von Normalarbeitszeit abzugeben, die an einer weitverbreiteten Praxis von Arbeitszeitgestaltung ansetzt, ohne die basalen Schutzfunktionen von Normalarbeitszeit aufgeben zu müssen.

\section{LITERATUR}

Backhaus, K./Erichson, B./Plinke, W./Weiber, R. (2003): Multivariate Analysemethoden. Eine anwendungsorientierte Einführung, Berlin Bauer, F./Groß H./Lehmann, L./Munz, E. (2004): Arbeitszeit 2003. Arbeitszeitgestaltung, Arbeitsorganisation und Tätigkeitsprofile, Berichte des ISO 70, Köln

Groß, H./Thoben, T./Bauer, F. (1989): Arbeitszeit '89. Ergebnisse einer aktuellen Repräsentativbefragung zu den Arbeitszeitstrukturen und Arbeitszeitwünschen der abhängig Beschäftigten in der Bundesrepublik Deutschland, in: Minister für Arbeit, Gesundheit und Soziales des Landes Nordrhein-Westfalen (Hrsg.): Arbeitszeit ‘89. Ein Report zu den Arbeitszeiten und Arbeitszeitwünschen der abhängig Beschäftigten in der Bundesrepublik, Düsseldorf

Groß, H./Schwarz, M. (2006): Betriebs- und Arbeitszeiten 2005. Ergebnisse einer repräsentativen Betriebsbefragung, Manuskript Herrmann, C./Promberger, M./Singer, S./Trinczek, R. (1999): Forcierte Arbeitszeitflexibilisierung, Berlin

Hinrichs, K. (1988): Motive und Interessen im Arbeitszeitkonflikt, Frankfurt/New York
Hosmer, D./Lemeshow, S. (1989): Apllied logistic regression, New York Janßen, D./Nachreiner, F. (2004): Flexible Arbeitszeiten, Dortmund/ Berlin/Dresden

Jirjahn, U. (2004): Welche Betriebe nutzen Schichtarbeit?, in: Bellmann, L./Schnabel,C. (Hrsg.): Betriebliche Arbeitszeitpolitik im Wandel, BeitrAB 288, Nürnberg, S. 67-85

Kurz-Scherf, I. (1995): Zeit der Vielfalt - Vielfalt der Zeiten. Individuelle und betriebliche Arbeitszeiten und Arbeitszeitpräferenzen in Berlin. Schriftenreihe der Senatsverwaltung für Frauen und Arbeit, Bd. 11, Berlin Schank, T./Schnabel, C. (2004): Betriebliche Determinanten des Überstundeneinsatzes, in: Bellmann, L./Schnabel, C. (Hrsg.): Betriebliche Arbeitszeitpolitik im Wandel, BeitrAB 288, Nürnberg, S. 37-62 Seifert, H. (1995): Kriterien für eine sozialverträgliche Arbeitszeitgestaltung, in: Büssing, A./Seifert, H. (Hrsg.): Sozialverträgliche Arbeitszeitgestaltung, München und Mering, S. 15-30

Seifert, H. (2007): Arbeitszeit - Entwicklungen und Konflikte, in: Aus Politik und Zeitgeschichte 4-5, S. 17-24 